\title{
Present and future of cancer immunotherapy: A tumor microenvironmental perspective (Review)
}

\author{
YU YU and JIUWEI CUI
}

\author{
Cancer Center, The First Hospital of Jilin University, Changchun, Jilin 130021, P.R. China
}

Received September 21, 2017; Accepted June 26, 2018

DOI: $10.3892 / \mathrm{ol} .2018 .9219$

\begin{abstract}
Modulation of the tumor microenvironment is becoming an increasingly popular research topic in the field of immunotherapy, and studies regarding immune checkpoint blockades and cancer immunotherapy have pushed cancer immunotherapy to a climax. Simultaneously, the manipulation of the immune regulatory pathway can create an effective immunotherapy strategy; however, the tumor microenvironment serves an important role in suppressing the antitumor immunity by its significant heterogeneity. A number of patients with cancer do not have a good response to monotherapy approaches; therefore, combination strategies are required to achieve optimal therapeutic benefits. Targeting the tumor microenvironment may provide a novel strategy for immunotherapy, break down the resistance of conventional cancer therapy and produce the foundation for personalized precision medicine. The present review summarized the research regarding cancer immunotherapy from the perspective of how the tumor microenvironment affects the immune response, with the aim of proposing a novel strategy for cancer immunotherapy and combination therapy.
\end{abstract}

\section{Contents}

1. Introduction

2. Formation and development of the tumor immunosuppressive microenvironment

3. Heterogeneity of the tumor microenvironment

4. Immunotherapies targeted to the tumor microenvironment

5. Conclusions and perspectives

Correspondence to: Professor Jiuwei Cui, Cancer Center, The First Hospital of Jilin University, 71 Xinmin Street, Changchun, Jilin 130021, P.R. China

E-mail: cuijw@jlu.edu.cn

Key words: tumor microenvironment, cancer immunotherapy, immune checkpoint blockade, combination therapy

\section{Introduction}

Cancer immunotherapy is emerging as a beneficial tool for cancer treatment by activating the immune system to produce antitumor effects (1). Recently, cancer immunotherapy, particularly immune checkpoint therapy, has progressed and provided novel strategies for the treatment of cancer. The most advanced approach to therapeutically utilize the antitumor activity is via immune checkpoint inhibitors. This strategy has recently achieved notable clinical success in patients with numerous malignant cancer types; for example, in patients with advanced melanoma, the blockade of cytotoxic T lymphocyte-associated antigen 4 (CTLA-4) via the antibody ipilimumab and the inhibition of the programmed death 1 (PD-1) receptor via the antibody nivolumab resulted in improved overall survival time $(2,3)$. In comparison to conventional therapies for cancer, including radiation and chemotherapy, cancer immunotherapy primarily targets the immune system or tumor microenvironment rather than tumor cells themselves, and can induce a synergistic effect in combination therapies (4). However, the efficacy of cancer immunotherapy is limited to only certain patients, due to not all patients responding to these immunomodulatory maneuvers, and there are notable differences between individuals (5). The manner in which to improve the efficacy of patients with cancer is fast becoming the focus of cancer immunotherapy.

There is increasing evidence demonstrating that the differences in the outcome of cancer immunotherapy are attributed to the heterogeneity of the tumor microenvironment (6). The tumor microenvironment consists of tumor cells, tumor-infiltrating immune cells, cancer-associated fibroblasts (CAFs), the tumor vasculature and the extracellular matrix (ECM), which collectively can promote tumor transformation, protect the tumor from host immunity, support tumor growth and invasion, foster therapeutic resistance and provide niches for dormant metastases to thrive (7). The presence of malignant tumor cells initiates a series of changes that can transform the tumor environment into one that can promote cancer progression. The orchestration of these changes involves recruitment and activation of CAFs, migration of immune cells, stroma remodeling, development of tumor vascular networks, upregulation of the suppressive receptors on tumor cells and reprogramming of cell metabolism (8). The complexity of these changes results in the heterogeneity of the tumor microenvironment. Furthermore, the tumor immunosuppressive microenvironment is formed 
with the development of tumor proliferation and the increasing heterogeneity of the tumor microenvironment, which may influence the cancer immunotherapy.

In the present review, the progression of tumor microenvironment heterogeneity, its development and the effect on immunotherapy, and the present and future of cancer immunotherapy from the perspective of the tumor microenvironment are summarized.

\section{Formation and development of the tumor immuno- suppressive microenvironment}

Although tumor cells initially instigate the formation of the tumor microenvironment, the mutual influence and co-evolution among tumor cells, stroma components and immune cells continuously promote the development of immunosuppressive progress (7). Tumor cells utilize the negative regulatory mechanism of the immune system, in order to establish a full range of immunosuppressive states in the tumor microenvironment, which will create the conditions for their survival and development (9).

Effects of tumor cells on the tumor microenvironment. Tumor cells promote immune escape by forming an immunosuppressive microenvironment. Antigens expressed on the surface of tumor cells are usually in a defective state. The decreased or absent expression of major histocompatibility complex class I restricts the activation of the tumor-infiltrating lymphocytes (TILs) (9). The existing suppressive signal transduction in the immune system could be utilized by tumor cells, including PD-1, ligand programmed death-ligand 1 (PD-L1), CTLA-4, cytotoxic T lymphocyte activation gene 3 (LAG-3), T cell immunoglobulin domain 3 mucin domain protein 3 (Tim-3) and cluster of differentiation (CD)160 (10), which may gradually result in $\mathrm{T}$ cell exhaustion (11); therefore, the inhibited function of TILs in the tumor microenvironment results in tumor immunosuppression. Tumor cells can also secrete immunosuppressive factors, including transforming growth factor- $\beta$ (TGF- $\beta$ ), interleukin-6 (IL-6), IL-10, vascular endothelial growth factor (VEGF) and matrix metalloproteinase $(5,9)$, in order to cause tumor-infiltrating immune cells to inhibit their antitumor effect.

Furthermore, abnormal metabolism of tumor cells can also enhance the immunosuppressive effect of the tumor microenvironment. Normal cells acquire energy primarily through oxidative phosphorylation, and a limited number use glycolysis, which can be inhibited under aerobic conditions; however, the method by which tumor cells acquire energy is different, and is termed aerobic glycolysis or 'the Warburg effect' (12). In this condition, tumor cells maintain an increased rate of glycolysis even in the presence of adequate oxygen. This was initially considered to be a strategy to adapt to hypoxia, but it is now widely accepted that this shift of energy metabolism is not only to produce the necessary resources for the biosynthetic activities of tumor cells, but also to generate numerous acidic products to form the acidic tumor microenvironment, which results in immunosuppression (13). In addition to aerobic glycolysis, in order to rapidly proliferate, tumor cells are also required to increase the demand for amino acids. Among them, glutamine, methionine, tryptophan, arginine and leucine are essential for the tumor cells as metabolic regulators in supporting cancer cell growth (14), and the tumor cells are more competitive for these metabolic resources compared to tumor-infiltrating immune cells. Additionally, indoleamine 2,3-dioxygenase (IDO), which is highly expressed by tumor cells, is a rate-limiting enzyme of tryptophan metabolism that has regulatory effects on $\mathrm{T}$ cells resulting from tryptophan depletion in tumor microenvironments (15). In addition, the tryptophan metabolites, including 3-hydroxyquinolinic acid via the kynurenine pathway, can also directly inhibit $\mathrm{T}$ effector cells (16). Furthermore, the metabolic interplay between tumor cells and immune cells can contribute to the exhaustion of TILs and immunosuppression (17).

Effect of CAF on the tumor microenvironment. Fibroblasts are the dominant component of the tumor stroma (18). The important functions of fibroblasts include deposition of ECM, regulation of epithelial differentiation, regulation of inflammation and participation in wound healing. Activated fibroblasts, termed CAFs, are also critical for the formation of the tumor microenvironment, particularly for solid tumor types (19). Fibroblasts are a critical determinant in the tumor malignant progression and represent an important target for cancer therapies.

Fibroblast activation protein $\alpha(\mathrm{FAP} \alpha)$ is selectively expressed on the surface of CAF, and the majority of epithelial tumor types exhibit high expression of FAP $\alpha$ (20). FAP $\alpha$ has a dual role as a protease and in signal transduction. The former refers to its involvement in remodeling the construction of the microenvironment stroma by degrading fibronectin and changing the structure of collagen, in order to enhance the invasion ability of tumor cells along the fibers (21). The latter refers to its involvement with TGF- $\beta$, VEGF, stromal cell-derived factor-1, platelet-derived growth factor, hepatocyte growth factor and other cytokines, which could conduct signals to promote tumor growth, prevent immune cell recruitment, inhibit the function of tumor-infiltrating immune cells and enhance ECM proliferation for the formation of a tumor biological barrier (22). Furthermore, the desmoplastic stroma could then surround the tumor cells and prevent access of antitumor drugs (23). Kraman et al (24) confirmed that depleting FAP-expressing cells could reduce the occurrence of hypoxic necrosis in vitro and permit the immunological control of growth in vivo; therefore, FAP-expressing cells are an important immunosuppressive component of the tumor microenvironment.

Effect of the tumor abnormal vascular structure on the tumor microenvironment. Tumor angiogenesis is an important process in the tumor microenvironment. Emerging evidence indicates that angiogenesis and immunosuppression frequently occur simultaneously in response to different stimuli (25). Tumor neovascularization is primarily leaky, tortuous, dilated and saccular. The structural and functional abnormalities of tumor blood vessels result in the impaired blood supply and interstitial hypertension or high interstitial fluid pressure (IFP) (26). The perfusion of tumor tissues is further hampered by the formation of hypoxia and high IFP in the microenvironment of malignant tumor types. The imbalance between the promotion and inhibition of angiogenic factors contributes to 
the abnormal structure of the tumor vasculature (27). Among a whole range of pro-angiogenic factors that participate in physiological or pathological angiogenesis, VEGF is the most important and also a potent angiogenic factor that can increase the density of tumor blood vessels (28). The sufficient expression of VEGF depends on the oxygen concentration in tissues. There are a variety of transcription factors in tumor tissues, including hypoxia inducible factor (HIF), which can upregulate the VEGF under hypoxic conditions (29).

Therefore, combined with the abnormal metabolism of tumor cells, the suitable microenvironment for tumor survival is characterized by low $\mathrm{pH}$, hypoxia and high IFP, which is considered to aid in rendering tumor microenvironments hostile to the immune cells. The low $\mathrm{pH}$ or acidic microenvironment can accelerate the differentiation of regulatory $\mathrm{T}$ cells (Tregs) and the development of myeloid-derived suppressor cells by promoting the production of IL-2, inhibiting the infiltration of $\mathrm{T}$ cells and inducing their apoptosis, and activating tumor-associated macrophages (TAMs), in order to secrete a large number of cytokines to promote tumor angiogenesis (30). Hypoxia can promote the formation of tumor blood vessels by upregulating the expression of pro-angiogenic factors such as HIF-1, VEGF, IL-6, TNF $\alpha$, and tyrosine kinase receptor Tie2 (31), and increase the malignancy and trigger tumor metastasis by inducing epithelial-mesenchymal transition (EMT) (32). High IFP can prevent immune cells from recruiting to the tumor tissue and interfere with drug delivery. Additionally, high IFP and pro-angiogenic factors can also evoke lymphangiogenesis, which is the important physiological basis of lymphatic metastasis (26). Collectively, these vascular abnormalities result in a complex immunosuppressive microenvironment, which may promote the survival and metastasis of tumor cells.

\section{Heterogeneity of the tumor microenvironment}

The formation of the tumor immunosuppressive microenvironment is a dynamic and complex process. In addition to the significant heterogeneity of tumor cells, heterogeneity of stroma components and immune cells can also increase the complexity of the tumor microenvironment (8). Additionally, tumor progression, pathological stage, treatment efficacy and prognosis are also associated with the tumor microenvironment, which determines the antitumor response and remains a notable obstacle for the treatment of cancer (33). Therefore, due to the presence of tumor microenvironmental heterogeneity, the degree of the antitumor immune response in different individuals is variable.

The heterogeneity of stroma components in the tumor microenvironment is common. In pancreatic, breast and prostate cancer, and other solid tumor types with a high content of CAF, the formation of high-density ECM will increase the tumor IFP, and hinder the absorption of chemotherapy drugs and the infiltration of immune cells (19). Additionally, tumors with different types, locations and stages also exhibit tumor vascular heterogeneity (34). For instance, pancreatic ductal adenocarcinoma (PDA) is a stroma-rich cancer type and its tumor environment has been demonstrated to consist of an abundance of stroma containing numerous cells types, but predominantly pancreatic stellate cells (PSCs) (35). The vasculature in PDA is notably influenced by the excessive desmoplasia caused by the secretion of PSCs and finally leads to hypovascularity and perfusion impairment (36), which indicates that the role of tumor angiogenesis in the progression of pancreatic cancer is less notable compared with that of other hypervascular tumor types, including liver cancer. Kashiwagi et al (37) reported that murine melanoma cells were intracranially and subcutaneously implanted into mice, and the results determined that the vascular density of the intracranial tumor types was increased compared with that of subcutaneous tissues, but that the diameter was reduced. Compared with early renal cell carcinoma, advanced renal cell carcinoma exhibits an increased endothelial cell proliferation fraction, while presenting with a reduced microvessel density, which indicates that the heterogeneity in angiogenic activity is associated with tumor stage (38). Additionally, these structural abnormalities of tumor vasculature contribute to the spatial and temporal heterogeneity in tumor blood flow, and solid pressure generated by proliferating tumor cells compresses intratumor blood and lymphatic vessels, which further impairs not only the blood flow, but also the lymphatic flow (39); therefore, the heterogeneity in the stroma of tumor microenvironment requires consideration regarding the efficacy of immunotherapies.

The recruitment, differentiation and location of immune cells in the tumor microenvironment are variable among different tumor types, and their heterogeneity is also affected. Chevrier et al (40) conducted mass cytometry for high-dimensional single-cell analysis in order to produce an in-depth human atlas of the tumor immune microenvironment in patients with clear cell renal cell carcinoma, and this demonstrated the immune cell diversity in the tumor ecosystem and the fact that a number of specific immune signatures could function as biomarkers associated with progression-free survival. Additionally, the innate immune landscape in early lung adenocarcinoma indicates that the heterogeneity of immune cells may begin to form at an early tumor stage and evolve with the progression of the tumor stage, gradually compromising the antitumor immunity (41). Furthermore, the dominant types of immune cells infiltrated in tumor microenvironments are also different. TAMs are among the most frequently located cells in the pancreatic tumor microenvironment, while the majority of other tumor types are primarily dominated by TILs (42); therefore, the usage of agonist CD40 monoclonal antibody in PDA can activate and recruit a large number of macrophages, which are tumoricidal and could facilitate the depletion of the tumor stroma (43). The tumor microenvironment includes a complex network of immune T-cell subpopulations, and the state of activation, the location of infiltration and the density of the tumor stroma could be different (44). Therefore, due to the heterogeneity of immune cells in the tumor immune microenvironment, Chen et al (45) divided it into three phenotypes according to the distribution of immune cells, as follows: i) Immune-inflamed phenotype, where intratumor infiltration of $\mathrm{CD}^{+}$and $\mathrm{CD} 8^{+} \mathrm{T}$ cells, and parenchyma and stroma can be observed in a large number of immune cells; ii) immune-excluded phenotype, where immune cells cannot penetrate the parenchyma and only exist in the stroma; and iii) immune-desert phenotype, which a paucity of tumor-specific $\mathrm{T}$ cells are located in the parenchyma or 
stroma. Among them, the first two phenotypes are associated with non-inflamed tumor types. This classification explains the heterogeneity of immune cells in the tumor microenvironment, indicating that the tumor microenvironment can be modified by changing the immune phenotype, and providing a theoretical basis for personalized immunotherapy.

\section{Immunotherapies targeted to the tumor microenvironment}

The complexity and heterogeneity of the tumor immunosuppressive microenvironment increases the difficulty of cancer immunotherapy and is an important reason for the variable efficacy in immunotherapies. Unlike directly targeting the tumor cells, the tumor microenvironment represents an increasingly popular therapeutic target with a decreased risk of resistance and recurrence due to the genetically stable stromal cells (46). Recently, the immune checkpoint inhibitors have provided new hope and have become a focus of current cancer immunotherapy (47). The antitumor immunity points of the tumor microenvironment also have other beneficial targets, and application together with conventional cancer therapies can also provide a survival benefit for increased numbers of patients with cancer. The association between the heterogeneity of the tumor microenvironment and the main microenvironment-targeted therapies is demonstrated in Fig. 1; therefore, the combination or 'cocktail' therapy for cancer provides an increased number of advantages compared with monotherapy, and has become an important method to improve the efficacy of tumor immunotherapy (48).

Immune checkpoint blockade. Immune checkpoint inhibitors are strategies for activating immune function and normalizing the tumor microenvironment. Immune checkpoint inhibitors have become an effective means of treating numerous tumor types (49). The anti-PD-1/PD-L1 monoclonal antibody has been successfully used in clinical application and has already been approved for use in numerous cancer types, including melanoma, non-small cell lung cancer, kidney cancer and bladder cancer (50). Clinical trials are currently being used to determine the success of the application of the anti-PD-1 antibody (nivolumab) for different malignant tumor types, and the objective response rate (ORR) has been found to be variable: $32 \%$ of melanoma, $29 \%$ of renal cell carcinoma, $17 \%$ of non-small cell lung cancer (33\% of squamous cell carcinoma and $12 \%$ of non-squamous cell carcinoma) (51), and only $13.3 \%$ of head and neck cancer (52). The mechanism of inhibitor therapy is the activation of $\mathrm{T}$ cells, which requires an adequate number of TILs; therefore, the heterogeneity of the antitumor immune response is directly associated with the density of TILs. According to Teng et al (53), the tumor microenvironment could be stratified into four different types based on the presence or absence of TILs and PD-L1 expression, as follows: i) Type I (TILs $\left.{ }^{+} \mathrm{PD}-\mathrm{Ll}^{+}\right)$, where the tumor microenvironment is PD- $\mathrm{L1}^{+}$, with TILs driving adaptive immune resistance, indicating that it may benefit from a single-agent anti-PD-1/L1 blockade; ii) type II (TILs-PD-L1'), where the tumor microenvironment is PD-L1', with no TILs, indicating immune ignorance; iii) type III (TILs-PD-L1'), where the tumor microenvironment is PD-L1', with no TILs, indicating intrinsic induction and that the recruitment of
TILs is necessary; and iv) type IV (TILs ${ }^{+} \mathrm{PD}-\mathrm{L1}^{-}$), where the tumor microenvironment is PD-L1', with TILs, indicating the role of other suppressor pathways in promoting immune tolerance. For type II tumors, due to their inability to produce an antitumor immune response in the tumor microenvironment, the recruitment of $\mathrm{T}$ cells should be a priority. This stratification of the tumor microenvironment can predict the clinical efficacy of anti-PD-1/PD-L1 therapies and enable the optimal combination of cancer therapies tailored to target different tumor microenvironments (54). Furthermore, the latest research demonstrated that the expression of PD-L1 on the minimal residual disease would increase when the tumor recurs and acquires treatment resistance, while the proportion of effector cells could consistently express increased PD-1 and Tim-3 expression in the tumor microenvironment (55). This indicates that the expression of immune checkpoints should be monitored dynamically, and that the combination treatments may be valuable for improving efficacy and preventing recurrence in patients with tumors.

Recently, anti-PD-1 antibody Keytruda (pembrolizumab) has been approved by the FDA to treat solid tumor with microsatellite instability-high or mismatch repair-deficient (56). This approval confirms the important position of the immunotherapy-targeted tumor microenvironment in the cancer therapies and produces a foundation for cancer immunotherapy as a major part of the combination therapy strategy for different tumor types. With the success of PD-1/PD-L1 inhibitors in cancer immunotherapy, combination therapy with other immune checkpoints has received increasing attention in order to achieve greater clinical benefit. The combination therapies, including dual immune checkpoint inhibitors, are undergoing clinical trials. Clinical studies have demonstrated that the anti-PD-1/PD-L1 antibodies integrated with CTLA-4 inhibitors can increase the therapeutic efficacy and the percentage of responders in the treatment of advanced melanoma, indicating that the combination of immune checkpoint inhibitors can significantly enhance antitumor immunity (57). Currently, the indications and safety of PD-1/PD-L1 inhibitors in combination with CTLA-4 inhibitors for potential usage have also been investigated (58), and this combination was approved by the US Food and Drug Administration (FDA) for patients with BRAF V600 wild-type, unresectable or metastatic melanoma. Recently, a phase II clinical trial (CheckMate 069) indicated that the combination of first-line nivolumab plus ipilimumab could result in improved outcomes compared with first-line ipilimumab alone in patients with advanced melanoma, and the 2-year survival rates were 63.8 and $53.6 \%$, respectively (59). Additionally, Wei et al (60) demonstrated the distinct underlying mechanisms of anti-PD-1 and anti-CTLA-4 checkpoint blockade therapies using a mass cytometry-based systems approach to identify different subsets of exhausted T cells, which could improve the understanding of why the combination checkpoint blockade therapies are more effective than monotherapy. In other words, this combination could overcome the heterogeneity of TILs. Additionally, anti-PD-1/PD-L1 treatment can be used in combination with the inhibition of other immune checkpoints. Notably, the inhibition of PD-1 can stimulate other immune checkpoints expressed on $\mathrm{T}$ cells and increase the resistance of anti-PD-1 therapies, including Tim-3 (61,62) and LAG-3 (63), providing a theoretical basis for 


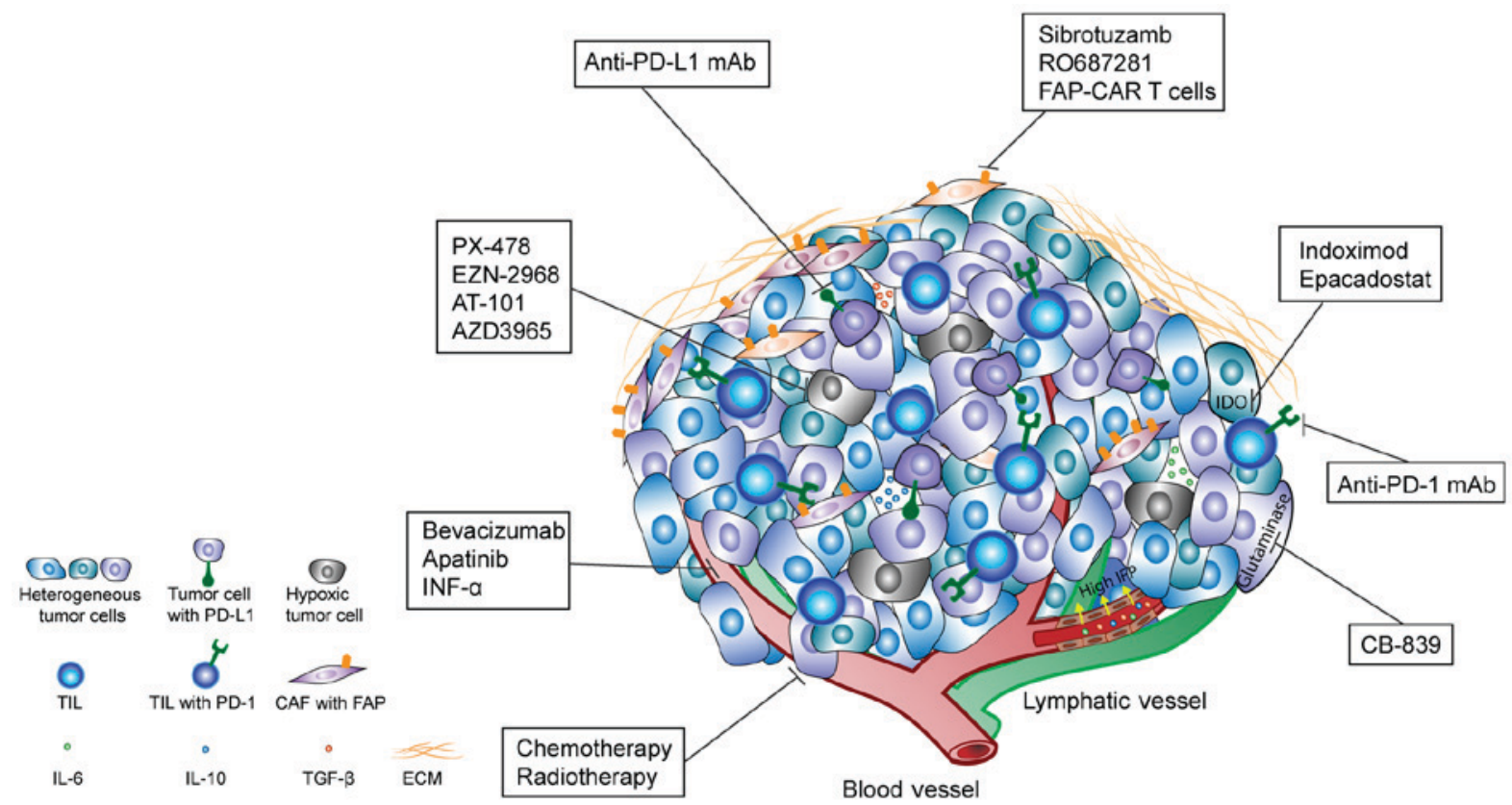

Figure 1. Heterogeneity of the tumor microenvironment and the main microenvironment-targeted therapies. Tumor cells, expression of biomarkers, oxygen concentration, $\mathrm{pH}$, IFP, angiogenesis, metabolism, ECM and other intra- and extra-tumor characters exhibit notable heterogeneity. Tumor cells can secrete factors into the ECM, including TGF- $\beta$, IL-6 and IL-10, in order to inhibit the function of TIL and result in tumor immunosuppression. These corresponding therapies include: Anti-PD-1 and anti-PD-L1 antibody targeting the immunosuppressive microenvironment; IDO inhibitor (epacadostat and indoximod) and glutaminase inhibitor (CB-839) targeting the tumor abnormal amino acids metabolism; hypoxia inducible factor 1 $\alpha$ inhibitors (PX-478 and EZN-2968), B-cell lymphoma 2 inhibitor (AT-101) and monocarboxylate transporter inhibitor (AZD3965) targeting the hypoxic tumor cells in the hypoxic tumor microenvironment; anti-angiogenic inhibitors (bevacizumab, INF- $\alpha$ and apatinib) and FAP $\alpha$ inhibitor (sibrotuzumab, RO6874281 and FAP-CAR T cells) targeting the regulation of tumor stroma; and combination therapies with chemotherapy, radiotherapy and other therapies. IFP, interstitial fluid pressure; TGF- $\beta$, transforming growth factor- $\beta$; IL, interleukin; PD-1, programmed death 1; PD-L1, PD-ligand 1; IDO, indoleamine 2,3-dioxygenase; INF- $\alpha$, interferon- $\alpha$; FAP, fibroblast activation protein; FAP-CAR, FAP-specific chimeric antigen receptor; TIL, tumor-infiltrating lymphocyte; CAF, cancer-associated fibroblast; ECM, extracellular matrix.

the combination therapy. Currently, all 5 clinical trials regarding the combined inhibition of Tim-3 and PD-1 are undergoing recruitment (NCT02817633, NCT03099109, NCT02608268, NCT03066648 and NCT02947165). Additionally, 8 clinical trials containing the combination of LAG-3 and PD-1 antibodies are also recruiting (NCT02658981, NCT01968109, NCT02061761,NCT03005782,NCT02676869,NCT02966548, NCT02488759 and NCT02060188) (https://clinicaltrials.gov/). Claudin-low breast cancer, an aggressive subtype that confers poor prognosis and exhibits a high expression level of EMT genes, has been reported to recruit Tregs to the tumor microenvironment, which inhibits an effective antitumor immune response (64), and this study indicated that future clinical trials should target the immunosuppressive elements in the tumor microenvironment in combination with immune checkpoint blockades in order to increase the efficacy.

However, the combinations of immunotherapies are not always successful. Two independent studies $(65,66)$ demonstrated that the concurrent administration of the anti-PD-1 antibody and the agonist antibody to OX40, a tumor necrosis factor family costimulatory receptor that could promote the activation and expansion of $\mathrm{T}$ cells, had an adverse effect on the antitumor response of OX40 stimulation and resulted in poor outcomes in mice. Additionally, the antitumor effect of sequential anti-OX40 and anti-PD-1 combination is controversial between the two studies; therefore, the sequence and timing of immunotherapies are critical to the success of combination therapy, and require further investigation prior to clinical use.
The appropriate selection of immune checkpoints inhibitors or other immunotherapies for personalized combination therapy is an indispensable option and the underlying mechanism requires further investigation.

Tumor metabolism regulation. Improving the immunosuppression of the tumor microenvironment by regulating tumor metabolism is a popular research topic. Immunotherapy with inhibition of IDO to inhibit tumor metabolism has achieved notable results. Currently, there are two primary drug types directed against IDO: i) Highly potent IDO inhibitor that directly inhibits the degradation of tryptophan, such as the drug epacadostat (67); and ii) IDO pathway inhibitor that inhibits the degradation of tryptophan and also reverses IDO-mediated immune suppression, such as the drug indoximod (68). Additionally, the safety and clinical efficacy of these two drug types have also been confirmed in recent clinical trials $(69,70)$. Significant breakthroughs in the studies of tumor cell metabolism have also provided novel options to combine with immunotherapies. IDO inhibitor epacadostat and anti-PD-1 antibody pembrolizumab have been demonstrated to have a promising clinical efficacy and safety for advanced cancer types in clinical trials improved objective response rate and disease control rate (71). Furthermore, a clinical trial has been initiated to evaluate the preliminary efficacy of indoximod combined with immune checkpoint inhibitors (NCT02073123); therefore, it is possible to conclude that IDO inhibitors have a potential synergistic effect with 
immune checkpoint inhibitors. Additionally, due to the success of IDO inhibitors, other tumor-associated amino acids with abnormal metabolism are gaining increasing attention. For example, clinical trials of glutaminase inhibitor CB-839 alone (NCT02861300) and combined with nivolumab (NCT02771626) for the treatment of solid tumor types are also under recruitment.

Hypoxic and acidic microenvironments are associated with the consequence of tumor metabolism, and reversing them is also being used as a strategy to regulate the tumor microenvironment. PX-478 is a selective inhibitor that can suppress hypoxia-induced HIF-1 $\alpha$ levels (72). In a previous clinical trial, patients with refractory solid tumor types were treated with EZN-2968, a locked nucleic acid antisense oligonucleotide against HIF-1 $\alpha$ (73). The number of cases was too small to veritably reflect the efficacy, but even so, there were a number of patients who responded to the treatment. Additionally, lactate can specifically upregulate B-cell lymphoma 2 (Bcl-2) through translational control and can promote resistance to the glucose starvation of tumor cells (74). In the clinical trial of abnormal lactate metabolism, it was determined that the use of cisplatin and etoposide in combination with Bcl-2 inhibitor AT-101 could enhance the antitumor effect (75). Furthermore, the clinical trial regarding the the transport of lactic acid, pyruvate and other metabolites, and monocarboxylate transporter inhibitor AZD3965, which could prevent the release of lactic acid by hypoxic tumor cells and then inhibit their growth and survival, is also recruiting (NCT01791595) (76).

Tumor stroma regulation. Regulation of the heterogenous stroma components in the tumor microenvironment could modulate its immunosuppressive conditions. Promoting normalization of tumor blood vessels and weakening the function of CAFs are the key roles in effectively transporting oxygen, drugs or immune cells and other components to tumor tissues, reducing the tumor proliferation and invasion (77).

The first anti-angiogenic therapy, Avastin (bevacizumab), was approved by the FDA in 2003. Considerable effort into the development of anti-angiogenic therapies has been undertaken, and a number of these inhibitory agents have been approved for clinical use against a number of cancer types; however, tumors can frequently escape the effects of these agents, causing the disease to eventually progress (78). Therefore, anti-angiogenic therapy may serve a role in vessel normalization, in order to increase immune cell infiltration and enhance the efficacies of immunotherapies (79). The combined treatment of bevacizumab and interferon- $\alpha$ has also entered phase 2 and 3 clinical trials and demonstrated improved clinical efficacy in metastatic renal cell carcinoma, confirming the clinical value of combined application of anti-vascular therapy and immunotherapy when compared with monotherapy $(80,81)$. Furthermore, the clinical trials combined with VEGF receptor tyrosine kinase inhibitor apatinib and PD-1 inhibitor are currently recruiting, in order to evaluate the efficacy for the treatment of gastric cancer types (NCT03092895 and NCT02942329).

Additionally, there are several studies have also attempted to improve the immunosuppressive condition by modulating the function of CAFs in the tumor microenvironment. The humanized monoclonal antibody sibrotuzumab, which is directed against the specific antigen FAP $\alpha$ on
CAFs, could block its dual function of protease and signal transduction, then inhibit tumor progression, invasion and metastasis progression, and reduce its negative regulation of antitumor immunity. In phase 1 and 2 clinical trials using sibrotuzumab alone $(82,83)$, only a limited number of patients achieved stable disease and the expected clinical response rate was not met; however, whether the efficacy of the treatment could be improved by combining it with other immunotherapies requires further investigation. RO6874281 is a bispecific antibody containing an IL-2 variant targeting FAP $\alpha$. The IL-2 variant does not bind to Tregs, which could prevent the immunosuppressive capacity of the Tregs (84). By specifically targeting FAP $\alpha$, the antibody could not only increase the local IL-2 concentration, in order to activate the immune effector cells in the tumor microenvironment, but also inhibit the deterioration of the tumor microenvironment by directly blocking FAP $\alpha$. Due to the consistent expression of FAP in the tumor stroma, modified T cells that express a FAP-specific chimeric antigen receptor have also been engineered to inhibit the tumor proliferation and augment host immunity (85-87). Clinical trials regarding monotherapy (NCT02627274) and combination with other immunotherapies (NCT03063762) are currently being conducted to evaluate the safety, tolerability and preliminary therapeutic efficacy.

Therefore, studies regarding the stroma components in the tumor microenvironment will continue to be conducted in order to improve the effectiveness of the tumor immunosuppression and provide a novel alternative approach for personalized combination therapy.

Combination with chemotherapy and radiotherapy. Immunotherapy combined with traditional radiotherapy and chemotherapy has received increasing attention. Different chemotherapeutic drugs have different immunological mechanisms underlying the efficacy of the cancer therapy (88), including: i) Increased immunogenicity resulting in tumor cell apoptosis from drugs such as anthracycline, 5-fluorouracil (5-Fu) and oxaliplatin; ii) direct immunostimulation activating the tumor immunity of immune effector cells from such as gemcitabine, paclitaxel and pemetrexed; and iii) indirect immunostimulation inhibiting the immunosuppressive cells from drugs such as 5-Fu, cyclophosphamide and oxaliplatin. Additionally, radiotherapy can also influence the tumor immune response. Tumor cell death from irradiation can enhance the antitumor immunity by inducing antigen expression on tumor cells and activating lymphocytes $(89,90)$, and by generating the abscopal effect (91). Chemotherapy or radiotherapy can eliminate a number of the tumor cells in advance, then expose a large number of the tumor antigens and neoantigen products in the microenvironment, which could recruit increased numbers of immune effector cells, and finally improve the immunosuppressive state of the tumor microenvironment. Currently, the rationale for combining immunotherapy with chemotherapy and radiotherapy has been verified (92), and preclinical studies have also been well investigated $(93,94)$; therefore, it is plausible that combining immunotherapy with standard conventional therapies, including chemotherapy or radiotherapy, will provide synergistic antitumor effects (95), but 
the most beneficial dose and the appropriate time requires investigation $(89,96)$.

\section{Conclusions and perspectives}

The tumor immunosuppressive microenvironment is in a dynamic status and is coordinated by multiple immunosuppressive signals in the regulatory network. In the course of clinical cancer treatment, due to the tumor types, stages, histological features and other microenvironment-associated factors, heterogeneity of the tumor microenvironment will cause immunosuppression and then result in the differences in the efficacy of immunotherapies. Despite the success in targeting non-tumor cell components, including immune checkpoint blockade, focusing on a single immunosuppressive target is ineffective in the majority of patients with cancer. Even among the cancer types that do respond to the immune checkpoint inhibitors, including melanoma, non-small cell lung cancer and renal cell cancer, few patients exhibit objective control of tumor progression. Following blocking or inhibiting of one immunosuppressive signal, the tumor will compensate through other mechanisms to generate the resistance and reduce the efficacy of this immunotherapy. The association between heterogeneity of the tumor microenvironment and the immunotherapy response remains a significant challenge.

In the future, immunotherapy may be required to be tailored for each patient with cancer according to the tumor microenvironment. The application of novel immune biomarkers and the ability to monitor and evaluate the tumor microenvironment by novel strategies, in order to improve early cancer diagnosis and predict the therapeutic efficacy and prognosis, requires further investigation. Personalized immunotherapy based on individual genetic, molecular and immune profiling has the potential to produce the most optimized outcomes for patients with cancer, but healthcare costs must be kept in an affordable range. Notably, the combinations must be designed in a rational and safe manner, and further clinical trials should be conducted, in order to verify the combination therapies prior to progressing to clinical use.

\section{Acknowledgments}

Not applicable.

\section{Funding}

The present study was supported by the National Key Research and Development Program of China (grant no. 2016YFC1303800), the National Health and Family Planning Commission of China (grant no. ZX-07-C2016004), the Key Laboratory Construction Project of Science and Technology Department in Jilin Province (grant no. 20170622011JC), the Industrial Technology Research and Development Special Project of Development and Reform Commission in Jilin Province (grant no. 2017C022) and the Development and Reform Commission project in Jilin Province (grant no. 2014N147).

\section{Availability of data and materials}

Not applicable.

\section{Authors' contributions}

YY was responsible for the literature search and manuscript preparation. JC was responsible for study design, manuscript co-writing and correction. Both authors revised the article and approved the final version for publication.

\section{Ethics approval and consent to participate}

Not applicable.

\section{Patient consent for publication}

Not applicable.

\section{Competing interests}

The authors declare that they have no competing interests.

\section{References}

1. Whiteside TL, Demaria S, Rodriguez-Ruiz ME, Zarour HM and Melero I: Emerging opportunities and challenges in cancer immunotherapy. Clin Cancer Res 22: 1845-1855, 2016.

2. Hodi FS, O'Day SJ, McDermott DF, Weber RW, Sosman JA, Haanen JB, Gonzalez R, Robert C, Schadendorf D, Hassel JC, et al: Improved survival with ipilimumab in patients with metastatic melanoma. N Engl J Med 363: 711-723, 2010.

3. Topalian SL, Sznol M, McDermott DF, Kluger HM, Carvajal RD, Sharfman WH, Brahmer JR, Lawrence DP, Atkins MB, Powderly JD, et al: Survival, durable tumor remission, and long-term safety in patients with advanced melanoma receiving nivolumab. J Clin Oncol 32: 1020-1030, 2014.

4. Tang H, Qiao J and Fu YX: Immunotherapy and tumor microenvironment. Cancer Lett 370: 85-90, 2016.

5. Beatty GL and Gladney WL: Immune escape mechanisms as a guide for cancer immunotherapy. Clin Cancer Res 21: 687-692, 2015.

6. Klemm F and Joyce JA: Microenvironmental regulation of therapeutic response in cancer. Trends Cell Biol 25: 198-213, 2015

7. Swartz MA, Iida N, Roberts EW, Sangaletti S, Wong MH, Yull FE, Coussens LM and DeClerck YA: Tumor microenvironment complexity: Emerging roles in cancer therapy. Cancer Res 72: 2473-2480, 2012.

8. Junttila MR and de Sauvage FJ: Influence of tumour micro-environment heterogeneity on therapeutic response. Nature 501: 346-354, 2013.

9. Becker JC, Andersen MH, Schrama D and Thor Straten P: Immune-suppressive properties of the tumor microenvironment. Cancer Immunol Immunother 62: 1137-1148, 2013.

10. Chen L and Flies DB: Molecular mechanisms of T cell co-stimulation and co-inhibition. Nat Rev Immunol 13: 227-242, 2013.

11. Wherry EJ: T cell exhaustion. Nat Immunol 12: 492-499, 2011.

12. Warburg O, Wind F and Negelein E: The metabolism of tumors in the body. J Gen Physiol 8: 519-530, 1927.

13. Vander Heiden MG, Cantley LC and Thompson CB: Understanding the Warburg effect: The metabolic requirements of cell proliferation. Science 324: 1029-1033, 2009.

14. Walls J, Sinclair L and Finlay D: Nutrient sensing, signal transduction and immune responses. Semin Immunol 28: 396-407, 2016.

15. Fallarino F, Grohmann U, Vacca C, Bianchi R, Orabona C, Spreca A, Fioretti MC and Puccetti P: T cell apoptosis by tryptophan catabolism. Cell Death Differ 9: 1069-1077, 2002.

16. Munn DH and Mellor AL: Indoleamine 2,3 dioxygenase and metabolic control of immune responses. Trends Immunol 34: 137-143, 2013.

17. Jiang Y, Li Y and Zhu B: T-cell exhaustion in the tumor microenvironment. Cell Death Dis 6: e1792, 2015.

18. Kalluri R and Zeisberg M: Fibroblasts in cancer. Nat Rev Cancer 6: 392-401, 2006.

19. Kalluri R: The biology and function of fibroblasts in cancer. Nat Rev Cancer 16: 582-598, 2016. 
20. Fearon DT: The carcinoma-associated fibroblast expressing fibroblast activation protein and escape from immune surveillance. Cancer Immunol Res 2: 187-193, 2014.

21. Lee HO, Mullins SR, Franco-Barraza J, Valianou M, Cukierman E and Cheng JD: FAP-overexpressing fibroblasts produce an extracellular matrix that enhances invasive velocity and directionality of pancreatic cancer cells. BMC Cancer 11: $245,2011$.

22. Shimoda M, Mellody KT and Orimo A: Carcinoma-associated fibroblasts are a rate-limiting determinant for tumour progression. Semin Cell Dev Biol 21: 19-25, 2010.

23. Rucki AA and Zheng L: Pancreatic cancer stroma: Understanding biology leads to new therapeutic strategies. World J Gastroenterol 20: 2237-2246, 2014.

24. Kraman M, Bambrough PJ, Arnold JN, Roberts EW, Magiera L, Jones JO, Gopinathan A, Tuveson DA and Fearon DT: Suppression of antitumor immunity by stromal cells expressing fibroblast activation protein-alpha. Science 330: 827-830, 2010.

25. Motz GT and Coukos G: The parallel lives of angiogenesis and immunosuppression: Cancer and other tales. Nat Rev Immunol 11: 702-711, 2011

26. Rofstad EK, Galappathi K and Mathiesen BS: Tumor interstitial fluid pressure-a link between tumor hypoxia, microvascular density, and lymph node metastasis. Neoplasia 16: 586-594, 2014.

27. Goel S, Duda DG, Xu L, Munn LL, Boucher Y, Fukumura D and Jain RK: Normalization of the vasculature for treatment of cancer and other diseases. Physiol Rev 91: 1071-1121, 2011.

28. Carmeliet P and Jain RK: Molecular mechanisms and clinical applications of angiogenesis. Nature 473: 298-307, 2011.

29. Shweiki D, Itin A, Soffer D and Keshet E: Vascular endothelial growth factor induced by hypoxia may mediate hypoxia-initiated angiogenesis. Nature 359: 843-845, 1992.

30. Calcinotto A, Filipazzi P, Grioni M, Iero M, De Milito A, Ricupito A, Cova A, Canese R, Jachetti E, Rossetti M, et al: Modulation of microenvironment acidity reverses anergy in human and murine tumor-infiltrating $\mathrm{T}$ lymphocytes. Cancer Res 72: 2746-2756, 2012.

31. Balamurugan K: HIF-1 at the crossroads of hypoxia, inflammation, and cancer. Int J Cancer 138: 1058-1066, 2016.

32. Wu MZ, Tsai YP, Yang MH, Huang CH, Chang SY, Chang CC, Teng SC and Wu KJ: Interplay between HDAC 3 and WDR5 is essential for hypoxia-induced epithelial-mesenchymal transition. Mol Cell 43: 811-822, 2011.

33. Pottier C, Wheatherspoon A, Roncarati P, Longuespée R, Herfs M, Duray A, Delvenne P and Quatresooz P: The importance of the tumor microenvironment in the therapeutic management of cancer. Expert Rev Anticancer Ther 15: 943-954, 2015.

34. Jain RK: Normalizing tumor microenvironment to treat cancer: Bench to bedside to biomarkers. J Clin Oncol 31: 2205-2218, 2013.

35. Apte M, Pirola RC and Wilson JS: Pancreatic stellate cell: Physiologic role, role in fibrosis and cancer. Curr Opin Gastroenterol 31: 416-423, 2015.

36. Feig C, Gopinathan A, Neesse A, Chan DS, Cook N and Tuveson DA: The pancreas cancer microenvironment. Clin Cancer Res 18: 4266-4276, 2012.

37. Kashiwagi S, Izumi Y, Gohongi T, Demou ZN, Xu L, Huang PL, Buerk DG, Munn LL, Jain RK and Fukumura D: NO mediates mural cell recruitment and vessel morphogenesis in murine melanomas and tissue-engineered blood vessels. J Clin Invest 115: 1816-1827, 2005.

38. Baldewijns MM, Thijssen VL, Van den Eynden GG, Van Laere SJ, Bluekens AM, Roskams T, van Poppel H, De Bruine AP, Griffioen AW and Vermeulen PB: High-grade clear cell renal cell carcinoma has a higher angiogenic activity than low-grade renal cell carcinoma based on histomorphological quantification and qRT-PCR mRNA expression profile. Br J Cancer 96: 1888-1895, 2007.

39. Padera TP, Stoll BR, Tooredman JB, Capen D, di Tomaso E and Jain RK: Pathology: Cancer cells compress intratumour vessels. Nature 427: 695, 2004.

40. Chevrier S, Levine JH, Zanotelli VRT, Silina K, Schulz D, Bacac M, Ries CH, Ailles L, Jewett MAS, Moch H, et al: An immune atlas of clear cell renal cell carcinoma. Cell 169: 736-749.e18, 2017.

41. Lavin Y, Kobayashi S, Leader A, Amir ED, Elefant N, Bigenwald C, Remark R, Sweeney R, Becker CD, Levine JH, et al: Innate immune landscape in early lung adenocarcinoma by paired single-cell analyses. Cell 169: 750-765.e17, 2017.

42. Mielgo A and Schmid MC: Impact of tumour associated macrophages in pancreatic cancer. BMB Rep 46: 131-138, 2013.
43. Beatty GL,Chiorean EG,FishmanMP,Saboury B, Teitelbaum UR, Sun W, Huhn RD, Song W, Li D, Sharp LL, et al: CD40 agonists alter tumor stroma and show efficacy against pancreatic carcinoma in mice and humans. Science 331: 1612-1616, 2011.

44. Tosolini M, Kirilovsky A, Mlecnik B, Fredriksen T, Mauger S, Bindea G, Berger A, Bruneval P, Fridman WH, Pages F, et al: Clinical impact of different classes of infiltrating T cytotoxic and helper cells (Th1, th2, treg, th17) in patients with colorectal cancer. Cancer Res 71: 1263-1271, 2011.

45. Chen DS and Mellman I: Elements of cancer immunity and the cancer-immune set point. Nature 541: 321-330, 2017.

46. Quail DF and Joyce JA: Microenvironmental regulation of tumor progression and metastasis. Nat Med 19: 1423-1437, 2013.

47. Li Y, Li F, Jiang F, Lv X, Zhang R, Lu A and Zhang G: A Mini-review for cancer immunotherapy: Molecular understanding of PD-1/PD-L1 pathway \& translational blockade of immune checkpoints. Int J Mol Sci 17: pii: E1151, 2016.

48. Ledford H: Cocktails for cancer with a measure of immunotherapy. Nature 532: 162-164, 2016.

49. Sharma P and Allison JP: The future of immune checkpoint therapy. Science 348: 56-61, 2015.

50. Page DB, Bourla AB, Daniyan A, Naidoo J, Smith E, Smith M, Friedman C, Khalil DN, Funt S, Shoushtari AN, et al: Tumor immunology and cancer immunotherapy: Summary of the 2014 SITC primer. J Immunother Cancer 3: 25, 2015.

51. Gunturi A and McDermott DF: Nivolumab for the treatment of cancer. Expert Opin Investig Drugs 24: 253-260, 2015.

52. Ferris RL, Blumenschein G Jr, Fayette J, Guigay J, Colevas AD, Licitra L, Harrington K, Kasper S, Vokes EE, Even C, et al: Nivolumab for recurrent squamous-cell carcinoma of the head and neck. N Engl J Med 375: 1856-1867, 2016.

53. Teng MW, Ngiow SF, Ribas A and Smyth MJ: Classifying cancers based on t-cell infiltration and pd-11. Cancer Res 75: 2139-2145, 2015.

54. Smyth MJ, Ngiow SF, Ribas A and Teng MW: Combination cancer immunotherapies tailored to the tumour microenvironment. Nat Rev Clin Oncol 13: 143-158, 2016.

55. Kottke T, Evgin L, Shim KG, Rommelfanger D, Boisgerault N, Zaidi S, Diaz RM, Thompson J, Ilett E, Coffey M, et al: Subversion of NK-cell and TNFa immune surveillance drives tumor recurrence. Cancer Immunol Res 5: 1029-1045, 2017.

56. U.S. Food and drug administration: Treatment approved for Any Solid tumor with biomarker. Onco Times 39: 52-53, 2017.

57. Baumeister SH, Freeman GJ, Dranoff G and Sharpe AH: Coinhibitory pathways in immunotherapy for cancer. Annu Rev Immunol 34: 539-573, 2016.

58. Boutros C, Tarhini A, Routier E, Lambotte O, Ladurie FL, Carbonnel F, Izzeddine H, Marabelle A, Champiat S, Berdelou A, et al: Safety profiles of anti-CTLA-4 and anti-PD-1 antibodies alone and in combination. Nat Rev Clin Oncol 13: 473-486, 2016.

59. Hodi FS, Chesney J, Pavlick AC, Robert C, Grossmann KF, McDermott DF, Linette GP, Meyer N, Giguere JK, Agarwala SS, et al: Combined nivolumab and ipilimumab versus ipilimumab alone in patients with advanced melanoma: 2-year overall survival outcomes in a multicentre, randomised, controlled, phase 2 trial. Lancet Oncol 17: 1558-1568, 2016.

60. Wei SC, Levine JH, Cogdill AP,Zhao Y, Anang NAS, Andrews MC, Sharma P, Wang J, Wargo JA, Pe'er D and Allison JP: Distinct cellular mechanisms underlie anti-CTLA-4 and anti-PD-1 checkpoint blockade. Cell 170: 1120-1133.e17, 2017.

61. Shayan G, Srivastava R, Li J, Schmitt N, Kane LP and Ferris RL: Adaptive resistance to anti-PD1 therapy by Tim-3 upregulation is mediated by the PI3K-Akt pathway in head and neck cancer. Oncoimmunology 6: e1261779, 2016.

62. Koyama S, Akbay EA, Li YY, Herter-Sprie GS, Buczkowski KA, Richards WG, Gandhi L, Redig AJ, Rodig SJ, Asahina H, et al: Adaptive resistance to therapeutic PD-1 blockade is associated with upregulation of alternative immune checkpoints. Nat Commun 7: 10501, 2016.

63. Foy SP, Sennino B, dela Cruz T, Cote JJ, Gordon EJ, Kemp F, Xavier V, Franzusoff A, Rountree RB and Mandl SJ: Poxvirus-based active immunotherapy with PD-1 and LAG-3 dual immune checkpoint inhibition overcomes compensatory immune regulation, Yielding complete tumor regression in mice. PLoS One 11: e0150084, 2016.

64. Taylor NA, Vick SC, Iglesia MD, Brickey WJ, Midkiff BR, McKinnon KP, Reisdorf S, Anders CK, Carey LA, Parker JS, et al: Treg depletion potentiates checkpoint inhibition in claudin-low breast cancer. J Clin Invest 127: 3472-3483, 2017. 
65. Messenheimer DJ, Jensen SM, Afentoulis ME, Wegmann KW, Feng Z, Friedman DJ, Gough MJ, Urba WJ and Fox BA: Timing of PD-1 blockade is critical to effective combination immunotherapy with anti-OX40. Clin Cancer Res 23: 6165-6177, 2017.

66. Shrimali RK, Ahmad S, Verma V, Zeng P, Ananth S, Gaur P, Gittelman RM, Yusko E, Sanders C, Robins H, et al: Concurrent PD-1 blockade negates the effects of OX40 agonist antibody in combination immunotherapy through inducing T-cell apoptosis. Cancer Immunol Res 5: 755-766, 2017.

67. Yue EW, Sparks R, Polam P, Modi D, Douty B, Wayland B, Glass B, Takvorian A, Glenn J, Zhu W, et al: INCB24360 (Epacadostat), a highly potent and selective indoleamine-2,3-dioxygenase 1 (IDO1) inhibitor for immuno-oncology. ACS Med Chem Lett 8: 486-491, 2017.

68. Zhai L, Spranger S, Binder DC, Gritsina G, Lauing KL, Giles FJ and Wainwright DA: Molecular pathways: Targeting IDO1 and other tryptophan dioxygenases for cancer immunotherapy. Clin Cancer Res 21: 5427-5433, 2015.

69. Beatty GL, O'Dwyer PJ, Clark J, Shi JG, Bowman KJ, Scherle PA Newton RC, Schaub R, Maleski J, Leopold L, et al: First-in-human phase I study of the oral inhibitor of indoleamine 2,3-dioxygenase-1 epacadostat (INCB024360) in patients with advanced solid malignancies. Clin Cancer Res 23: 3269-3276, 2017.

70. Soliman HH, Minton SE, Han HS, Ismail-Khan R, Neuger A, Khambati F, Noyes D, Lush R, Chiappori AA, Roberts JD, et al: A phase I study of indoximod in patients with advanced malignancies. Oncotarget 7: 22928-22938, 2016.

71. Gangadhar TC, Hamid O, Smith DC, Bauer TM, Wasser JS Luke JJ, Balmanoukian AS, Kaufman DR, Zhao Y, Maleski J, et al: Preliminary results from a Phase I/II study of epacadostat (incb024360) in combination with pembrolizumab in patients with selected advanced cancers. J Immunother Cancer 3 (Suppl 2): O7, 2015

72. Zhu Y, Zang Y, Zhao F, Li Z, Zhang J, Fang L, Li M, Xing L, $\mathrm{Xu} \mathrm{Z}$ and $\mathrm{Yu}$ J: Inhibition of HIF-1 $\alpha$ by PX-478 suppresses tumor growth of esophageal squamous cell cancer in vitro and in vivo. Am J Cancer Res 7: 1198-1212, 2017.

73. Jeong W, Rapisarda A, Park SR, Kinders RJ, Chen A, Melillo G, Turkbey B, Steinberg SM, Choyke P, Doroshow JH, et al: Pilot trial of EZN-2968, an antisense oligonucleotide inhibitor of hypoxia-inducible factor-1 alpha (HIF-1 $\alpha$ ), in patients with refractory solid tumors. Cancer Chemother Pharmacol 73: 343-348, 2014

74. Huang C, Sheng S, Li R, Sun X, Liu J and Huang G: Lactate promotes resistance to glucose starvation via upregulation of Bcl-2 mediated by mTOR activation. Oncol Rep 33: 875-884, 2015.

75. Schelman WR, Mohammed TA, Traynor AM, Kolesar JM, Marnocha RM, Eickhoff J, Keppen M, Alberti DB, Wilding G, Takebe N and Liu G: A phase I study of AT-101 with cisplatin and etoposide in patients with advanced solid tumors with an expanded cohort in extensive-stage small cell lung cancer. Invest New Drugs 32: 295-302, 2014.

76. Kershaw S, Cummings J, Morris K, Tugwood J and Dive C: Optimisation of immunofluorescence methods to determine MCT1 and MCT4 expression in circulating tumour cells. BMC Cancer 15: 387, 2015.

77. Tarallo V and De Falco S: The vascular endothelial growth factors and receptors family: Up to now the only target for anti-angiogenesis therapy. Int J Biochem Cell Bio 64: 185-189, 2015.

78. Bueno MJ, Mouron S and Quintela-Fandino M: Personalising and targeting antiangiogenic resistance: A complex and multifactorial approach. Br J Cancer 116: 1119-1125, 2017.

79. Tian L, Goldstein A, Wang H, Ching Lo H, Sun Kim I, Welte T, Sheng K, Dobrolecki LE, Zhang X, Putluri N, et al: Mutual regulation of tumour vessel normalization and immunostimulatory reprogramming. Nature 544: 250-254, 2017.

80. Melichar B, Bracarda S, Matveev V, Alekseev B, Ivanov S, Zyryanov A, Janciauskiene R, Fernebro E, Mulders P Osborne $\mathrm{S}$, et al: A multinational phase II trial of bevacizumab with low-dose interferon- $\alpha 2 \mathrm{a}$ as first-line treatment of metastatic renal cell carcinoma: BEVLiN. Ann Oncol 24: 2396-2402, 2013.
81. Rini BI, Bellmunt J, Clancy J, Wang K, Niethammer AG, Hariharan $\mathrm{S}$ and Escudier B: Randomized phase III trial of temsirolimus and bevacizumab versus interferon alfa and bevacizumab in metastatic renal cell carcinoma: INTORACT trial. J Clin Oncol 32: 752-759, 2014.

82. Scott AM, Wiseman G, Welt S, Adjei A, Lee FT, Hopkins W, Divgi CR, Hanson LH, Mitchell P, Gansen DN, et al: A phase I dose-escalation study of sibrotuzumab in patients with advanced or metastatic fibroblast activation protein-positive cancer. Clin Cancer Res 9: 1639-1647, 2003.

83. Hofheinz RD, al-Batran SE, Hartmann F, Hartung G, Jäger D, Renner C, Tanswell P, Kunz U, Amelsberg A, Kuthan H and Stehle G: Stromal antigen targeting by a humanised monoclonal antibody: An early phase II trial of sibrotuzumab in patients with metastatic colorectal cancer. Onkologie 26: 44-48, 2003.

84. Arenas-Ramirez N, Woytschak J and Boyman O: Interleukin-2: Biology, design and application. Trends Immunol 36: 763-777, 2015.

85. Kakarla S, Chow KK, Mata M, Shaffer DR, Song XT, Wu MF, Liu H, Wang LL, Rowley DR, Pfizenmaier K, et al: Antitumor effects of chimeric receptor engineered human $\mathrm{T}$ cells directed to tumor stroma. Mol Ther 21: 1611-1620, 2013.

86. Tran E, Chinnasamy D, Yu Z, Morgan RA, Lee CC, Restifo NP and Rosenberg SA: Immune targeting of fibroblast activation protein triggers recognition of multipotent bone marrow stromal cells and cachexia. J Exp Med 210: 1125-1135, 2013

87. Wang LC, Lo A, Scholler J, Sun J, Majumdar RS, Kapoor V, Antzis M, Cotner CE, Johnson LA, Durham AC, et al: Targeting fibroblast activation protein in tumor stroma with chimeric antigen receptor T cells can inhibit tumor growth and augment host immunity without severe toxicity. Cancer Immunol Res 2: 154-166, 2014.

88. Galluzzi L, Zitvogel L and Kroemer G: Immunological mechanisms underneath the efficacy of cancer therapy. Cancer Immunol Res 4: 895-902, 2016.

89. Teng F, Kong L, Meng X, Yang J and Yu J: Radiotherapy combined with immune checkpoint blockade immunotherapy: Achievements and challenges. Cancer Lett 365: 23-29, 2015.

90. Kalbasi A, June $\mathrm{CH}$, Haas $\mathrm{N}$ and Vapiwala N: Radiation and immunotherapy: A synergistic combination. J Clin Invest 123 2756-2763,2013.

91. Park B, Yee C and Lee KM: The effect of radiation on the immune response to cancers. Int J Mol Sci 15: 927-943, 2014

92. Dalgleish AG: Rationale for combining immunotherapy with chemotherapy. Immunotherapy 7: 309-316, 2015.

93. Wargo JA, Reuben A, Cooper ZA, Oh KS and Sullivan RJ: Immune effects of chemotherapy, radiation, and targeted therapy and opportunities for combination with immunotherapy. Semin Oncol 42: 601-616, 2015.

94. Sharabi AB, Lim M, DeWeese TL and Drake CG: Radiation and checkpoint blockade immunotherapy: Radiosensitisation and potential mechanisms of synergy. Lancet Oncol 16: e498-e509, 2015.

95. Shahabi V,Postow MA,Tuck D and Wolchok JD: Immune-priming of the tumor microenvironment by radiotherapy: Rationale for combination with immunotherapy to improve anticancer efficacy. Am J Clin Oncol 38: 90-97, 2015.

96. Hughes PE, Caenepeel S and Wu LC: Targeted therapy and checkpoint immunotherapy combinations for the treatment of cancer. Trends Immunol 37: 462-476, 2016.

This work is licensed under a Creative Commons Attribution-NonCommercial-NoDerivatives 4.0 International (CC BY-NC-ND 4.0) License. 